\title{
Magnetic-resonance determination of the spatial dependence of the droplet size distribution in the cream layer of oil-in-water emulsions: Evidence for the effects of depletion flocculation
}

\author{
P. J. McDonald, ${ }^{1}$ E. Ciampi, ${ }^{1}$ J. L. Keddie, ${ }^{1}$ M. Heidenreich,${ }^{1,2}$ and R. Kimmich ${ }^{1,2}$ \\ ${ }^{1}$ Department of Physics, University of Surrey, Guildford, Surrey GU2 5XH, United Kingdom \\ ${ }^{2}$ Sektion Kernresonanzspektroskopie, Universität Ulm, Albert-Einstein-Allee 11, 89069 Ulm, Germany
}

(Received 14 July 1998)

\begin{abstract}
It is shown that a combination of pulsed-field-gradient spin-echo (PGSE) nuclear-magnetic-resonance (NMR) restricted diffusion analysis and NMR imaging may be used to measure the spatial dependence of the droplet size distribution in the cream layer of turbid oil-in-water emulsions. ${ }^{1} \mathrm{H}_{-}{ }^{13} \mathrm{C}$ cyclic $J$ cross-polarization PGSE is introduced as a technique for this purpose in cases where selective observation of the oil component (or other carbohydrate constituent) is required. With this method, ${ }^{13} \mathrm{C}$ nuclei are chemical shift selectively excited by cross-polarization from coupled ${ }^{1} \mathrm{H}$ partners. An optimum detection sensitivity is ensured by transferring the polarization back to the coupled protons with which the combined imaging and diffusion experiment is then carried out. The spatial dependence of the oil droplet size distribution was measured for a series of emulsions containing various fractions of gum xanthan thickener dissolved in the water. The experimental results are compared with a recent model of the creaming process due to Pinfield, Dickinson, and Povey [J. Colloid Interface Sci. 166, 363 (1994)]. When no gum xanthan is present, the experimental results are in good agreement with the model. However, the model fails to describe the droplet distribution for emulsions with a gum xanthan concentration of the order of $0.1 \mathrm{wt} \%$. The discrepancy is discussed in terms of depletion flocculation and depletion stabilization. [S1063-651X(99)10801-8]
\end{abstract}

PACS number(s): $61.25 . \mathrm{Hq}$

\section{INTRODUCTION}

The creaming of an emulsion, the gravimetric separation of the dispersed component from the continuous component, is an important technological process [1]. Gum xanthan, or similar water soluble polymers, are regularly used to increase the viscosity of the continuous component of an emulsion and thereby slow, and even inhibit, the creaming process. However, it has been shown that creaming may be accelerated by the addition of small fractions of gum xanthan to the continuous component of oil-in-water $(\mathrm{O} / \mathrm{W})$ emulsions stabilized with caseinate [2] and polyoxyethylene sorbitan oleate (a nonionic surfactant) [3]. This acceleration was attributed to depletion flocculation, which, for many years, has been recognized as a mechanism for flocculation-the joining together of particles-in colloidal systems where free macromolecules exist in the continuous medium [4].

In modeling emulsions, it is usually assumed that the creaming rate is determined by the Stokes' velocity of the droplets in the continuous component. Models vary in the way they treat acceleration to the Stokes' velocity, steric hindrance, and diffusion, but are otherwise broadly similar. The Stokes' velocity is proportional to the square of the droplet radius, and inversely proportional to the viscosity of the continuous component. Flocs have an effective size that is larger than that of the constituent particles, and consequently have a higher Stokes' velocity. On the other hand, higher concentrations of thickeners, such as gum xanthan, raise the viscosity of the aqueous medium and thereby retard the rate of creaming in an $\mathrm{O} / \mathrm{W}$ emulsion. Most existing models of creaming, such as that of Ref. [5], include the viscosity element in slowing creaming through calculation of the Stokes' velocity but do not explicitly consider the effect of time-dependent depletion flocculation in increasing the kinetics of the process. There is, however, a generally ac- cepted description of the mechanism of depletion flocculation as follows $[4,6]$. As two spherical particles in a medium containing free polymer molecules approach each other at distances less than twice the effective diameter of the molecules, there is a demixing of polymer segments and solvent which increases the Gibbs free energy of the system. On closer approach of the particles, expulsion of essentially pure solvent from between particles into the bulk polymer solution lowers the free energy. As a consequence, there is a potential barrier to particle approach, but, when it is exceeded, the particles are held together in a floc as a result of being in a potential minimum [1]. Monte Carlo simulations combined with the Flory-Huggins theory of polymer solutions have found that the height of the potential maximum and the depth of the potential minimum are similar in magnitude and can be of the order of a few $k T$ 's at room temperature, with both being proportional to particle size in a unimodal dispersion or emulsion. At the very lowest concentrations of the free polymer, the potential minimum is insufficiently deep to maintain flocs of particles. At slightly higher concentrations, the minimum is deep enough and the barrier to flocculation still sufficiently low that a significant fraction of particles overcome it and flocculate. At still greater concentrations of the free polymer, the barrier is higher and most particles fail to overcome it. Flocculation is then prevented by a mechanism referred to as depletion stabilization.

Creaming has, of course, long been studied with various noninvasive techniques, including ultrasound and light scattering [1], microscopy [7] and stray-field magnetic-resonance imaging [8]. However, the available experimental techniques to date have not been able to measure the droplet size distribution in turbid creaming emulsions as a function of position. Measurement of the spatial variation of the droplet size distribution is expected to provide evidence for the suggested 
effects of flocculation. Also, such a measurement would test more rigorously than hitherto possible the accuracy of existing models of creaming where flocculation is not important, and would reveal the limitations of the models where it is important.

The objective of this study is therefore twofold. First, we demonstrate that the spatial dependence of the droplet size distribution in the cream layer of oil-in-water emulsions may be measured using a combination of ${ }^{1} \mathrm{H}$ and ${ }^{13} \mathrm{C}$ edited nuclear magnetic resonance (NMR) imaging and pulsedfield-gradient spin-echo (PGSE) NMR diffusometry. This carries forward previous, nonspatially resolved and non- ${ }^{13} \mathrm{C}$ selective PGSE studies of stable emulsions [9,10]. Second, we show how the addition of gum xanthan to the emulsion changes the distribution, and compare it to model predictions. Being able to measure and then control the droplet size distribution in a cream layer offers an opportunity to enhance the technology of gravimetric separation used in food, cosmetics, agrochemical, and pharmaceutical industries.

PGSE NMR probes the microstructure of the emulsion droplets through restricted diffusion phenomena. Parameters of the droplet size distribution are evaluated from echo attenuation curves, and are mapped across the sample using magnetic resonance imaging. The primary limitation of using ${ }^{1} \mathrm{H}$ PGSE NMR for this purpose is that one is never totally sure that the residual water and, when it is at high concentration, surfactant, in the cream layer is not contributing significantly to the observed signal. For this reason we introduce a technique, the proton detected ${ }^{13} \mathrm{C}$ cyclic $J$ crosspolarization [11-13] (CYCLCROP) PGSE diffusometry. Employing CYCLCROP permits selective imaging and diffusometry experiments of specific $\mathrm{CH}_{N}$ groups to be carried out. The method therefore permits the unambiguous detection of the oil component of the emulsion. We refer to the combined imaging CYCLCROP PGSE method as CYCLCROP ${ }^{13} \mathrm{C}$ magnetic resonance mapping of droplet size parameters.

In this paper, the experimentally determined droplet size distributions of various emulsions containing increasing fractions of gum xanthan - and hence emulsions in which depletion flocculation might be increasingly important-are compared with the predictions of the model of the creaming process due to Pinfield, Dickinson, and Povey [5]. Incidental to the study is the observation of the creaming process itself, to be reported elsewhere, and an investigation of possible departure of the droplet shape from spherical in the cream layer.

\section{SAMPLES AND METHODS}

\section{A. Sample preparation}

A series of oil-in-water emulsions were prepared: one with a unimodal droplet size distribution and the rest with a bimodal distribution. The bimodal droplet size distribution amplified the effects of droplet size variation in the creaming process, and made the departure of the experimental data from the model more evident. The emulsions consist of 33.3wt $\%$ decane and 66.6-wt \% aqueous solution of gum xanthan (in various concentrations), and 1.0 -wt $\%$ nonionic surfactant, polyoxyethylene(20) sorbitan monooleate sold under the trade name of Tween 80 . In order to enhance the low
TABLE I. Characteristics of the prepared emulsions. (Dec, decane; Tol, toluene.)

\begin{tabular}{cccc}
\hline \hline Emulsion & $\begin{array}{c}\text { Oil } \\
(33.3 \% \text { of total })\end{array}$ & $\begin{array}{c}\text { Gum xanthan } \\
(\% \text { of water })\end{array}$ & $\begin{array}{c}\text { Droplet } \\
\text { distribution }\end{array}$ \\
\hline Ehom & $100 \%$ Dec & 0.100 & Unimodal \\
$E 100$ & $100 \%$ Dec/0\% Tol & 0.100 & Bimodal \\
$E 33$ & $80 \%$ Dec/20\% Tol & 0.033 & Bimodal \\
$E 10$ & $80 \%$ Dec/20\% Tol & 0.010 & Bimodal \\
$E 3$ & $80 \%$ Dec/20\% Tol & 0.003 & Bimodal \\
$E 0$ & $80 \% \mathrm{Dec} / 20 \% \mathrm{Tol}$ & 0.000 & Bimodal \\
\hline \hline
\end{tabular}

natural abundance of ${ }^{13} \mathrm{C}$, the decane in some of the emulsions was replaced by a mixture of decane and methyl ${ }^{13} \mathrm{C}$-enriched toluene in the ratio 80 parts decane to 20 parts toluene by weight. The toluene greatly improved the sensitivity of the ${ }^{13} \mathrm{C}$ edited NMR imaging.

Apart from the droplet size distribution, the primary variable distinguishing the emulsions is the concentration of gum xanthan. This varies between $0.00 \%$ and $0.10 \%$ by weight of the water fraction of the emulsion (Table I). The emulsion Ehom was prepared with a single droplet size distribution using an Ultra Turrax T8 (IKA Labortechnik, Germany) homogenizer. The remaining emulsions all have a bimodal droplet size distribution. They were prepared as follows. The oil was added to the aqueous solution, and the mixture stirred for 15 min using a magnetic stirrer and shaken in as reproducible fashion as possible for $5 \mathrm{~min}$. After this period of crude homogenization, the emulsion was divided into two equal parts, one of which was further homogenized using the Ultra Turrax T8 homogenizer for $1.25 \mathrm{~h}$. The two halves were then remixed. The various emulsion compositions are summarized in Table I.

The droplet size distribution was measured separately in the shaken and well homogenized emulsion fractions by analysis of optical micrographs. In common with much previous work $[1-3,9,10]$ it was found that the size distribution of both emulsions was well described by a log-normal size distribution function

$$
P(r)=\frac{A}{\sqrt{2 \pi} r \sigma} \exp \left(-\frac{\left(\ln \left(r / r_{0}\right)\right)^{2}}{2 \sigma^{2}}\right),
$$

where $r$ is the droplet radius, $r_{0}$ is the median radius, and $\sigma$ is a (dimensionless) measure of the width of the distribution. The prefactor $A$ was chosen so that the normalized volume fraction droplet size distribution of the separate emulsions was 0.5 . Figure 1 shows the optical data for emulsion $E 100$, which was typical of all the emulsions studied, together with log-normal fits. For the shaken emulsion the fit parameters are $A=2.93 \times 10^{-4}, r_{0}=7.95 \mu \mathrm{m}$, and $\sigma=0.522$, and, for the homogenized emulsion, $A=1.35 \times 10^{-2}, r_{0}=2.23 \mu \mathrm{m}$, and $\sigma=0.517$. The inset to the figure shows the volume fraction as a function of droplet size for the combined bimodal emulsion based on these fit parameters. A clear maximum in the volume distribution is seen around $3 \mu \mathrm{m}$, with a distinct break of gradient and long tail extending above $10 \mu \mathrm{m}$. It is to this volume distribution, rather than the number distribution, that the NMR signal intensities are most obviously sensitive. 


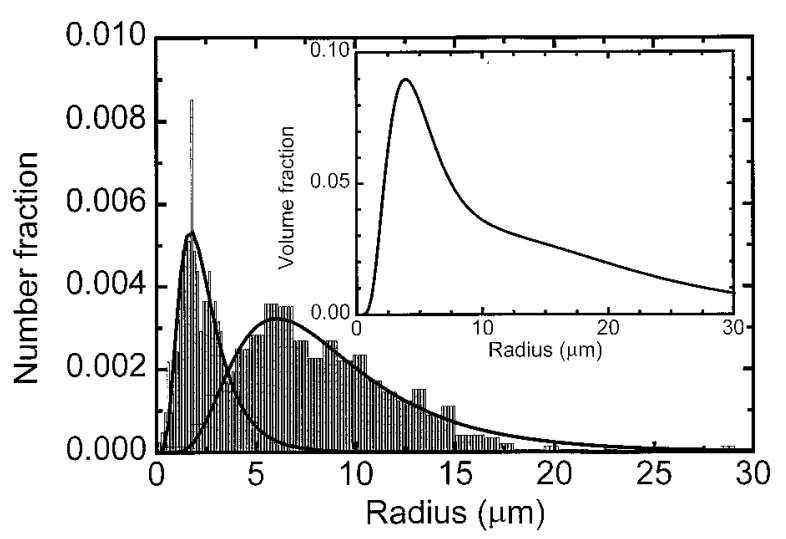

FIG. 1. The optically measured droplet size distribution for the two parts of emulsion E100 which is typical of them all. Two histograms are overlaid and fitted with log-normal distribution functions (see the text for parameters). The distribution with the larger droplets is shown $\times 100$. The inset shows the corresponding volume distribution for the combined emulsion.

\section{B. NMR experimental methods}

All the NMR experiments were carried out on a Bruker DSX400 NMR spectrometer with a ${ }^{1} \mathrm{H}$ NMR frequency of $400 \mathrm{MHz}$. The spectrometer was equipped with a 23-mm internal diameter radio frequency excitation-detection coil doubly tuned to both ${ }^{1} \mathrm{H}$ and ${ }^{13} \mathrm{C}$. The emulsions, typically $13 \mathrm{~mm}$ deep, were contained in small sealed pots $20 \mathrm{~mm}$ in diameter.

Self diffusion of ${ }^{1} \mathrm{H}$ was measured using the stimulatedecho variant of the PGSE technique [14-16]. Following excitation of the nuclear spin magnetization by a $90^{\circ}$ radio frequency pulse, a pulse of magnetic field gradient $\mathbf{G}$ is applied to the sample for a short encoding interval $\delta$. This pulse imposes a precessional phase shift on the nuclear spins dependent on position. It is followed at time $\tau_{1}$ after the first $90^{\circ}$ pulse by a second $90^{\circ}$ pulse which stores the magnetization along the $z$ axis for a period $\tau_{2}$. The magnetization is then recalled by a third $90^{\circ}$ pulse, whereupon a second identical gradient pulse is applied which imposes a further phase shift. An echo signal is formed, the magnitude of which is dependent on the diffusion occurring in the "diffusion interval" $\Delta$ between the two gradient pulses, as discussed in Sec. III. The stimulated echo sequence allows a longer diffusion time to be used than is the case with a direct echo sequence, where the diffusion time is limited by the short $T_{2}$ of the oil in the cream layer. Long times are required in order to observe fully the effects of restricted diffusion in the largest emulsion droplets. Experiments with pulse sequences designed to compensate for internal magnetic field gradients did not prove useful. Internal gradients turned out to be of negligible importance.

${ }^{1} \mathrm{H}$ self diffusion weighted profiles were acquired by prepending the PGSE experiment to a standard spin-echo profiling experiment with a single read gradient [16] [see Fig. 2(a)]. Indirectly detected ${ }^{13} \mathrm{C}$ self diffusion profiles were obtained using cyclic $J$ cross-polarization [11,12]. CYCLCROP involves the selection of ${ }^{13} \mathrm{C}$ satellites of ${ }^{1} \mathrm{H}$ nuclei found in a specific ${ }^{13} \mathrm{CH}_{N}$ molecular group while completely suppressing all noncoupled or unselected ${ }^{1} \mathrm{H}$. This highly selective spectral editing is achieved by two con- secutive cross-polarization processes in which magnetization is transferred within the selected molecular group from the ${ }^{1} \mathrm{H}$ to the $J$-coupled ${ }^{13} \mathrm{C}$ nucleus and back to the ${ }^{1} \mathrm{H}$ nuclei [see Fig. 2(b)]. To saturate all noncoupled protons, ${ }^{1} \mathrm{H}$ radio frequency and gradient pulses are applied while the desired magnetization is fully transferred (and stored) to the ${ }^{13} \mathrm{C} \mathrm{nu}-$ clei. In this way, one is certain of the origin of the ${ }^{1} \mathrm{H}$ signal, and in particular can suppress the signal due to water extremely well. For selective mapping of self diffusion within the oil, the initial $90^{\circ}$ excitation pulse of the PGSE profiling experiment is replaced by the CYCLCROP editing module. In this work, polarization transfer was achieved with the pulsed rotating frame transfer sequence with windows (PRAWN) variant of the experiment [13]. For PRAWN, the cross-polarization sequence consists of $n$ radio frequency pulses of length $\tau_{w}$ and flip angle $\alpha$ separated one from the other by $\tau_{s}$. The conditions for optimum transfer on resonance are that $\gamma_{\mathrm{C}} B_{1, \mathrm{C}}=\gamma_{\mathrm{H}} B_{1, \mathrm{H}}$ during the contact pulses where $\gamma_{\mathrm{C} ; \mathrm{H}}$ and $B_{1, \mathrm{C} ; \mathrm{H}}$ are the ${ }^{13} \mathrm{C}$ and ${ }^{1} \mathrm{H}$ magnetogyric ratios and radio frequency field strengths, respectively, that $n \alpha=2 \pi$, and that (for $\mathrm{CH}_{2}$ groups) $n\left(\tau_{w}+\tau_{s}\right)=(\sqrt{2} J)^{-1}$.

Self diffusion profiles were acquired for all the emulsions using a variety of gradient and timing parameters in order to measure the droplet size distribution as a function of position. Typical stimulated echo timing parameters used for the diffusion measurement are $\Delta=640 \mathrm{~ms}$ and $\delta=1 \mathrm{~ms}$, with $\tau_{1}=2 \mathrm{~ms}$. The diffusion gradient strength was the principal variable, although some measurements, not reported here, were made with a constant gradient and variable encoding time $\delta$. In the variable gradient strength measurements, the gradient strength was incremented between $G=0$ and 90 $\mathrm{G} / \mathrm{cm}$ in approximately $1-\mathrm{G} / \mathrm{cm}$ steps and was applied (with one exception discussed below) in the transverse $x$ direction. The profile spin-echo time was $2 \mathrm{~ms}$ and the read gradient strength was $11.4 \mathrm{G} / \mathrm{cm}$. The read gradient was applied, and hence the profile obtained, in the vertical $z$ direction. Figure 2 summarizes the pulse sequences used for ${ }^{1} \mathrm{H}$ and ${ }^{13} \mathrm{C}$ edited PGSE profiling, and more rigorously defines the timing parameters.

In addition to diffusion, the spatial dependence of $T_{1}$ was measured across each of the creamed emulsions using a saturation-recovery spin-echo profiling technique, with recovery times ranging from $0.1 \mathrm{~ms}$ to $10 \mathrm{~s}$. $T_{2}$ was also measured as a function of position across the emulsion. $T_{2}$ measurements were made using a spin-echo technique with variable echo time. For both $T_{1}$ and $T_{2}$, the imaging parameters are the same as for the self diffusion measurements.

The NMR measurements described here required between a few seconds, for a simple ${ }^{1} \mathrm{H}$ density profile, and a few hours, for a complete ${ }^{13} \mathrm{C}$ edited PGSE profiling analysis of droplet size with natural abundance ${ }^{13} \mathrm{C}$. The ${ }^{1} \mathrm{H}$ and enriched ${ }^{13} \mathrm{C}$ droplet size analyses typically required between 15 and $60 \mathrm{~min}$, dependent on the number of averages and number of gradient strengths acquired.

In every case, some three weeks was allowed to ensure that creaming of the emulsion was complete before the measurements were made. Moreover, comparison was made with data recorded after just four or five days.

\section{THEORY}

\section{A. Restricted diffusion PGSE attenuation}

For stationary nuclear spins, the phase shifts due to the two pulses of the magnetic field gradient in the PGSE ex- 


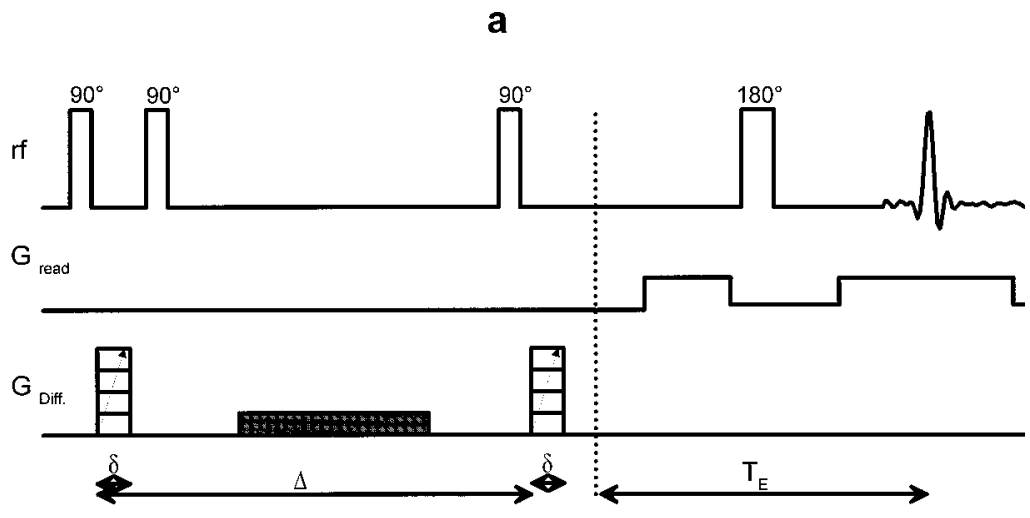

b
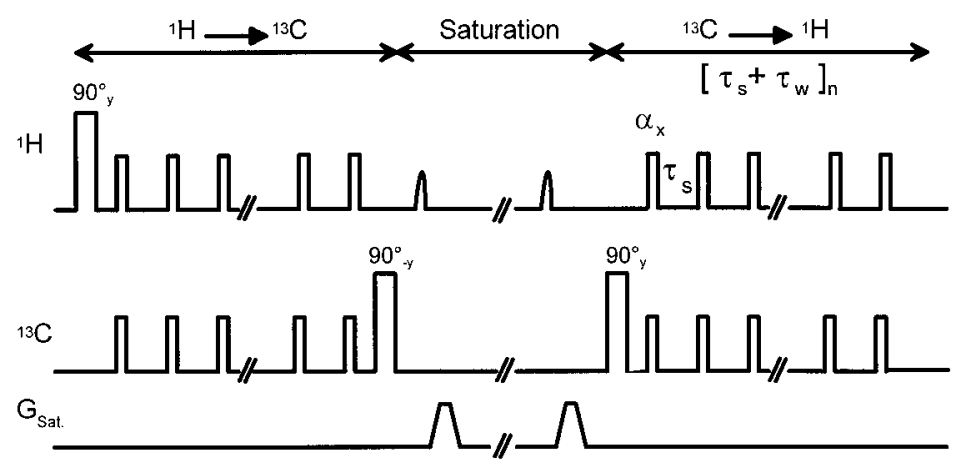

FIG. 2. (a) The pulse sequence used for the ${ }^{1} \mathrm{H}$ PGSE profiling experiments. (b) The CYCLCROP editing sequence which was prepended to the PGSE profiling sequence in place of the initial excitation pulse for the ${ }^{13} \mathrm{C}$ edited droplet size determinations. periment cancel. However, for diffusing spins a net phase shift is retained which, averaged over all spins in the sample, leads to an echo signal attenuation dependent on the diffusion coefficient $D$, gradient strength $G$, and timing parameters $\Delta$ and $\delta$. In the absence of restricted diffusion, the echo signal intensity is expected to vary as [16]

$$
E(\Delta, \delta, G)=E_{0} \exp \left(-\gamma^{2} G^{2} D \delta^{2}(\Delta-\delta / 3)\right)
$$

where $\gamma$ is the magnetogyric ratio of the nuclei, and $E_{0}$ is the $T_{2}$ weighted zero-gradient intensity. In essence, droplet sizes are calculated from a determination of $D$ as a function of $\Delta$, the "diffusion time." For short diffusion times, defined such that

$$
\Delta \ll \frac{\left\langle r^{2}\right\rangle}{6 D_{\text {bulk }}},
$$

where $r$ is the droplet radius, the oil diffuses in an unre- stricted manner and the measured $D$ equals the standard value, $D_{\text {bulk }}$. For longer times,

$$
\Delta \approx \frac{\left\langle r^{2}\right\rangle}{6 D_{\text {bulk }}}
$$

the maximum distance the oil can diffuse is limited by the droplet size, and so the measured $D$ varies with the inverse of the measurement time. In this way, through diffusion, the NMR signal is made sensitive to the microstructure of the sample.

The literature contains a number of references to the analysis of restricted diffusion in spheres of radius $r$. With time, the presented analyses have become increasingly exact but at the same time increasingly complex. Murday and Cotts [17] presented an early discussion which has been used widely in the analysis of emulsion diffusion data. According to Murday and Cotts, the echo attenuation is given by

$$
\begin{aligned}
E(\Delta, \delta, G, r)= & E_{0} \exp \left(-2 \gamma^{2} G^{2} \sum_{m=1}^{\infty} \frac{1}{\alpha_{m}^{2}\left(\alpha_{m}^{2} r^{2}-2\right)}\right. \\
& \left.\times\left[\frac{2 \delta}{\alpha_{m}^{2} D}-\frac{2+\exp \left(-\alpha_{m}^{2} D(\Delta-\delta)\right)-2 \exp \left(-\alpha_{m}^{2} D \Delta\right)-2 \exp \left(-\alpha_{m}^{2} D \delta\right)+\exp \left(-\alpha_{m}^{2} D(\Delta+\delta)\right)}{\alpha_{m}^{4} D^{2}}\right]\right)
\end{aligned}
$$


where $\alpha_{m}$ is the $m$ th root of the transcendental equation

$$
\frac{1}{\alpha r} J_{3 / 2}(\alpha r)=J_{5 / 2}(\alpha r),
$$

and $J_{m}(x)$ is the Bessel function of order $m$. This analysis is valid over a wide range of diffusion times spanning from the short to the long time limit. However, the Murday-Cotts analysis assumes a Gaussian distribution of phase displacements, which, in practice, restricts the magnitude of the wave vector $\mathbf{q}=(2 \pi)^{-1} \gamma \mathbf{G} \delta$ to small values less than the reciprocal droplet size. This restriction makes it difficult to obtain data which are at the same time sensitive to both small and large droplet sizes.

Tanner and Stejskal [18] presented an alternative analysis which is exact in the long time limit. According to Tanner and Stejskal, the echo signal attenuation is given by

$$
E(\delta, G, r)=E_{0} \frac{9(\gamma G \delta r \cos (\gamma G \delta r)-\sin (\gamma G \delta r))^{2}}{(\gamma G \delta r)^{6}} .
$$

Since the long time limit is assumed, neither $D$ nor $\Delta$ appear in this result. This analysis, too, has been used to interpret emulsion data, and is identical to Eq. (5) in the appropriate limits. Finally, Callaghan [19] presented an exact result applicable over the full time range which also includes the effects of surface relaxation, not considered either by Murday and Cotts or Tanner and Stejskal. However, this analysis is considerably more complex than either of the other two, and does not lend itself readily to the analysis of experimental data.

A particularly useful extension of the Tanner and Stejskal result was made in Ref. [20]. Equation (7) is well approximated by

$$
E(\delta, G, r)=E_{0} \exp \left(-\gamma^{2} \delta^{2} G^{2} r^{2} / 5\right)
$$

over a broad range of attenuation factors. This form offers a mathematically tractable means of obtaining an expression for the echo attenuation given a Gaussian volume distribution of droplet sizes,

$$
P_{\nu}(r)=\frac{1}{\sqrt{2 \pi} \sigma_{\nu}} \exp \left(-\frac{\left(r-r_{\nu 0}\right)^{2}}{2 \sigma_{\nu}^{2}}\right),
$$

which is

$$
E\left(\delta, G, r_{\nu 0}, \sigma_{\nu}\right)=E_{0} \frac{1}{\sqrt{1+2 \sigma_{\nu}^{2} \beta^{2}}} \exp \left(-\frac{\beta^{2} r_{\nu 0}^{2}}{1+2 \sigma_{\nu}^{2} \beta^{2}}\right),
$$

where $\beta^{2}=\gamma^{2} \delta^{2} G^{2} / 5$.

\section{B. Cream layer droplet size distribution-modeling}

Several models of creaming in emulsions have been presented in the literature. Here we adopt the recent model of Ref. [5]. This model considers two main processes, buoyancy and diffusion.
The droplets are assumed to move upwards relative to the water layer at their terminal Stokes' velocity. A semiempirical correction factor is included for steric hindrance. Allowance is made for the downflow of water in calculating the actual upward velocity relative to laboratory coordinates. In an emulsion with a distribution of sizes, larger droplets rise more quickly than smaller droplets, and cause a large water downflow. Thus smaller droplets, while still rising relative to the water, can descend relative to the laboratory. The droplets are also assumed to randomly diffuse. Diffusion is fastest for the smallest droplets. The droplets continue to rise until a critical oil concentration is reached in the cream layer, after which they can rise no more. The critical concentration is often considered to be $64 \%$, which corresponds to random close packing of spheres of a single size [21]. In reality, the critical concentration is usually much greater, since small droplets can occupy interstices between larger droplets and the droplets can compress. Experimental cream layer oil concentrations of the order of $90 \%$ have been measured.

According to the model, the flux of droplets of a given radius $r_{i}$ through a layer at height $z$ in the emulsion relative to the continuous component liquid can be expressed as

$$
J=u_{i} \phi_{i}-D_{i}^{m} \frac{\partial \phi_{i}}{\partial z},
$$

where $\phi_{i}$ is the local concentration of droplets of radius $r_{i}$, and $D_{i}^{m}$ is the mutual diffusion coefficient. Consequently, the local change in droplet density for droplets of this size is given by

$$
\frac{\partial \phi_{i}}{\partial t}=\frac{\partial}{\partial z}\left(-u_{i} \phi_{i}+D_{i}^{m} \frac{\partial \phi_{i}}{\partial z}\right)
$$

The velocity $u_{i}$ is given by

$$
u_{i}=\frac{2 r_{i}^{2}\left(\rho_{1}-\rho_{2}\right) g}{9 \eta} \frac{1-\phi}{(1+\phi)^{1 / 3} \exp (5 \phi(1-\phi) / 3)}-u_{f},
$$

where $\rho_{1}-\rho_{2}$ is the density difference of the two components, $\eta$ is the zero-shear rate viscosity of the continuous component, $g$ is the acceleration due to gravity, and $\phi=\Sigma \phi_{i}$ is the total oil concentration in the layer. The first term in Eq. (13) is the Stokes' velocity multiplied by the semiempirical hindrance factor, and $u_{f}$ is the continuous component liquid velocity in the opposite direction, given by

$$
u_{f}=\frac{\sum_{i} u_{i} \phi_{i}}{1-\Sigma_{i} \phi_{i}} .
$$

The diffusion coefficient is given by

$$
\begin{aligned}
D_{i}^{m}= & \frac{k T}{6 \pi \eta r_{i}} \frac{(1-\phi)}{(1+\phi)^{1 / 3} \exp (5 \phi(1-\phi) / 3)} \\
& \times\left(1+8 \phi+30 \phi^{2}\right)(1-\phi),
\end{aligned}
$$

where $T$ is the temperature and the factors are, in turn, the Stokes Einstein coefficient, the semiempirical concentration 


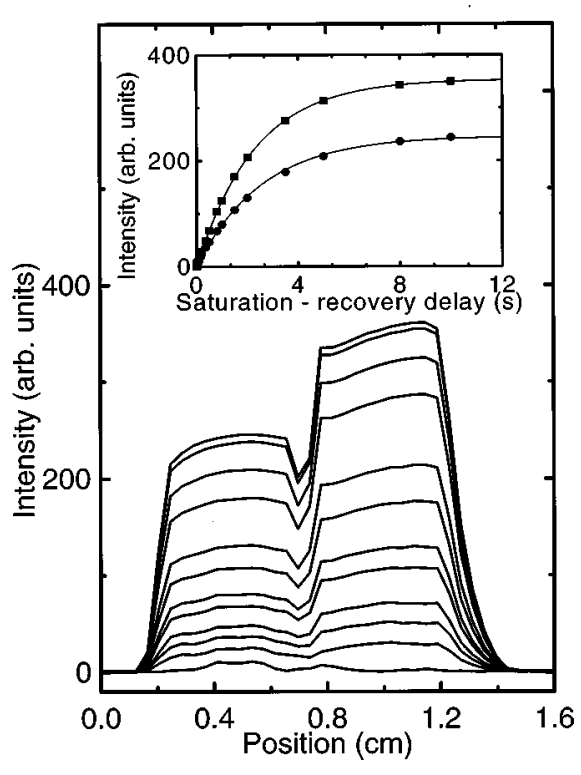

FIG. 3. Profiles of the creamed emulsion E0 recorded for saturation-recovery delays (from top) 10, 8, 5, 3.5, 2, 1.5, 1, 0.8, $0.5,0.35,0.2$, and $0.01 \mathrm{~s}$. The water layer extends from 0.2 to 0.75 $\mathrm{cm}$ on the scale, and the cream layer from 0.75 to $1.3 \mathrm{~cm}$. The inset shows the recovery curve (data points) and associated fit (solid curve) for the center of the water layer (circles, $T_{1}=2.58 \mathrm{~s}$ ) and cream layer (squares, $T_{1}=2.20 \mathrm{~s}$ ).

dependence correction coefficient, a term for excluded volume effects, and a fluid velocity correction.

Equation (12) is integrated numerically to yield the droplet size distribution as a function of time and space for each droplet size given appropriate boundary conditions. Expressing the model in this form makes clear the need to include a term involving $d u_{i} / d z$ as well as $d\left(\phi_{i}\right) / d z$ in the calculation. In our simulations, the initial distribution of droplets is assumed to be spatially uniform and-in terms of radius-to be that obtained from optical microscopy. At each stage of the calculation, the total droplet size distribution, which is used in calculating the hindrance factors, is evaluated by summing over droplets of all sizes. The calculation proceeds in a layer until the total concentration equals the critical concentration. For the emulsions studied here, the buoyancy term dominates the diffusion term. However, the latter has been included for completeness.

Although the model includes the viscosity of the continuous component, in practice it scales the buoyancy and diffusive terms in the same way. Consequently, although the absolute creaming rate varies with viscosity, the form of the droplet size distribution does not vary with the reduced time, $t^{\prime}=t / \eta$. The calculation need therefore only be performed once. It is clear that the model will be unable to explain differences in the spatial dependence of the droplet size distribution between measured emulsions, when the viscosity of the continuous component is the only variable parameter.

\section{RESULTS AND ANALYSIS}

\section{A. Spin-lattice $\left(T_{1}\right)$ relaxation}

The spin-lattice relaxation results are broadly the same for each emulsion, and the data for emulsion $E 0$ are presented here as typical of them all. Recovery profiles for a selection of recovery times are shown in Fig. 3. The profiles show the separated water to the left and the creamed oil layer to the right. The two regions are separated by a small gap which is the result of different average magnetic susceptibilities and chemical shifts in the water and cream layers. It is evident that $T_{1}$ is both long and spatially invariant in the cream and (as expected) water layers of the emulsion. Magnetization recovery curves for positions at the center of the water layer and the center of the cream layer are shown in the accompanying inset together with single component exponential recovery curve fits to the data. From these it is calculated that the water layer $T_{1}$ is $2.58 \pm 0.01 \mathrm{~s}$, and that the oil layer $T_{1}$ is $2.20 \pm 0.01 \mathrm{~s}$. No attempt has been made to fit a two component curve in the cream layer so as to include residual water separately: insufficient data are available. By common expectation, and as borne out by the experiments reported below, larger oil droplets are to be found predominantly toward the top of the cream layer. The fact that $T_{1}$ is long in the cream layer and does not depend on position (i.e., droplet size) suggests that surface relaxation is not an important process for oil in the emulsion droplets.

The ${ }^{1} \mathrm{H}$ signal intensity is significantly greater in the cream layer than in the separated water layer. This is surprising as the ${ }^{1} \mathrm{H}$ density of water is about $3.6 \%$ greater than that of the pure oil. The obvious conclusion that $T_{2}$ is substantially shorter than the echo time in the water layer is not supported by the $T_{2}$ measurements reported below, unless there is a very short $T_{2}$ component to the water layer not seen in these experiments. Such a short component could be associated with the surfactant and micelle formation. However, the surfactant concentration is very low. The discrepancy increases somewhat with gum xanthan concentration, but is not due entirely to the gum xanthan as the emulsion shown here is gum xanthan free. Moreover, the effect cannot be attributed to diffusive broadening in the imaging gradient. At worst, with no apparent diffusion in the cream layer due to confinement, this can only attenuate the water signal by about $4 \%$ relative to the oil for the experimental parameters used.

\section{B. Transverse $\left(T_{2}\right)$ relaxation}

The transverse relaxation results are also broadly similar for each of the emulsions, although they vary a little in detail. Typical $T_{2}$ decays taken from different positions within emulsion E0 are shown in Fig. 4. This is the emulsion for which $T_{2}$ is shortest and most variant across the cream layer. In the separated water layer the decays are monoexponential and $T_{2}$ is relatively long, $280 \pm 20 \mathrm{~ms}$. In the cream layer the data are also reasonably well represented by monoexponential decays, but $T_{2}$ is much shorter and varies significantly with position. It is almost $30 \mathrm{~ms}$ at the base of the cream layer. It drops rapidly to $11 \mathrm{~ms}$ near the center and rises again to about $18 \mathrm{~ms}$ at the very top. This change in $T_{2}$ with position is typical of all the bimodal emulsions, although the contrast between the minimum and maximum values is most marked in that with no xanthan. Indeed, the increase within the lower cream layer is barely observed in emulsion E100, where $T_{2}$ varies between $23 \mathrm{~ms}$ near the bottom and $30 \mathrm{~ms}$ at the top. Since the $T_{1}$ experiments suggest that surface relax- 


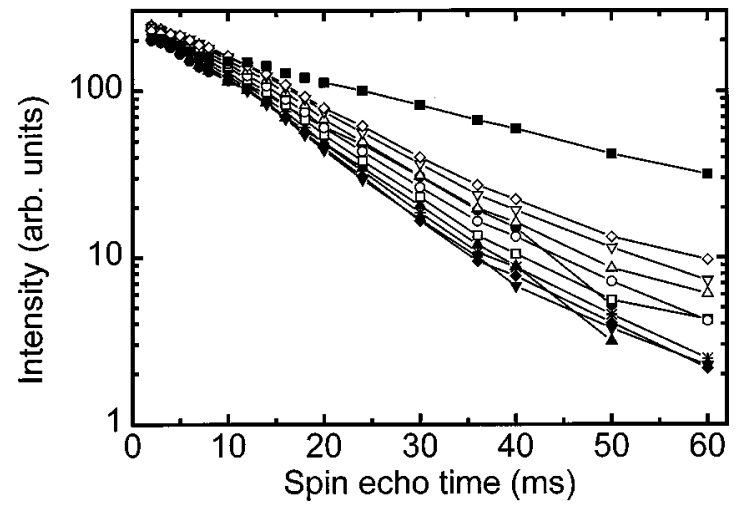

FIG. 4. Spin echo $T_{2}$ decay data for the cream layer of emulsion E0. Curves are shown in the order filled squares, circles, uptriangles, down-triangles, diamonds, stars, unfilled squares, circles, up-triangles, down-triangles, and diamonds, starting from the base of the cream and moving up in steps of $0.41 \mathrm{~mm}$. The $T_{2}$ values are $28.1,16.4,12.4,11.4,11.4,11.7,12.5,13.3,14.4,15.6$, and 16.6 $\mathrm{ms}$, respectively.

ation is not important for the oil droplets, the conclusion is that the shortening of $T_{2}$ in the cream layer is due to background magnetic field gradients arising from the magnetic susceptibility changes at the oil-water interfaces. The calculated $T_{2}$ therefore reflects, in an intangible manner, a complex averaging of field gradients due to droplets of different size distribution at different locations. Although the gradients due to magnetic susceptibility interfaces are much stronger for small $(1 \mu \mathrm{m})$ droplets, they also extend over many droplet diameters, and cancel to a significant degree. For larger droplets $(10 \mu \mathrm{m})$ the gradients are confined to near the droplet surfaces only.

\section{C. ${ }^{1} \mathrm{H}$ PGSE diffusometry}

Figures 5(a) and 5(b) show exemplar ${ }^{1} \mathrm{H}$ PGSE weighted profiles (every fifth profile is shown) for emulsions $E 0$ and $E 100$, respectively, recorded as a function of gradient

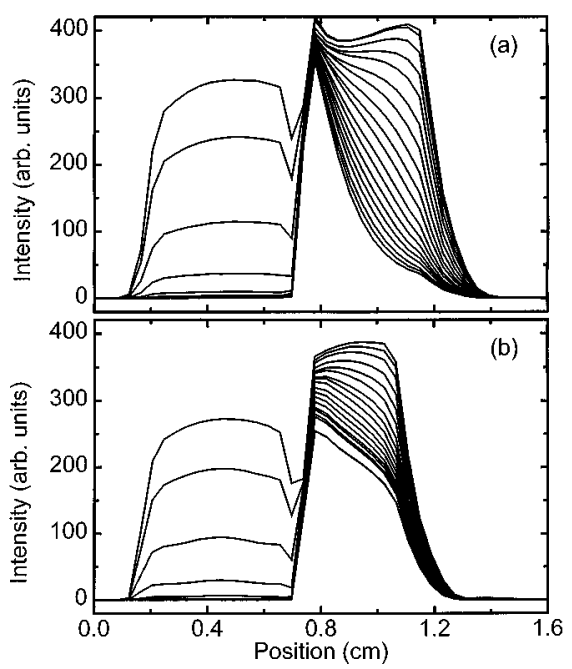

FIG. 5. (a) ${ }^{1} \mathrm{H}$ PGSE weighted profiles of creamed emulsion $E 0$. From the top, the profiles are shown for gradient strengths from 0.95 to $85.50 \mathrm{G} / \mathrm{cm}$ in steps of $4.75 \mathrm{G} / \mathrm{cm}$. (b) As in (a), but for emulsion $E 100$.

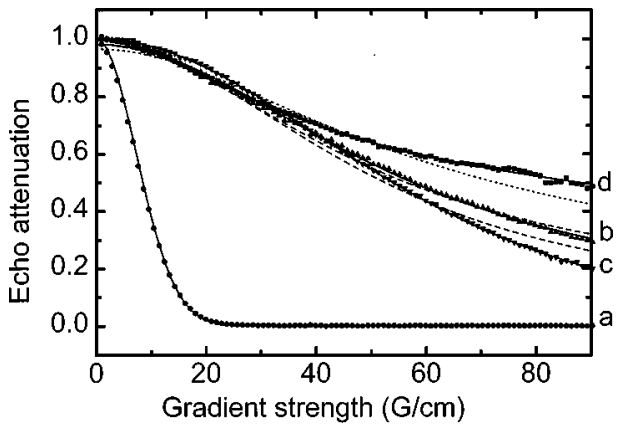

FIG. 6. (a) PGSE decay for the center of the water layer of emulsion E0 (circles) and an associated fit (solid line) according to Eq. (2). The diffusion coefficient of the water layer is $2.11 \times 10^{-5} \mathrm{~cm}^{2} \mathrm{~s}^{-1}$. (b) PGSE decay for the center of the cream layer (there is little discernible spatial variation) of emulsion Ehom (up-triangles) and associated fit (solid line) according to Eq. (10). The mean radius is $3.03 \mu \mathrm{m}$ and the standard deviation $2.64 \mu \mathrm{m}$. The lower and upper dashed lines are fits for the radius and the width, increased by $10 \%$ and $30 \%$, respectively. (c) PGSE decay for the center of the cream layer of emulsion E0 (down triangles) and associated fit (solid line). (d) PGSE decay for the center of the cream layer in emulsion $E 100$ (squares). The dotted line is a single component fit according to Eq. (10). The solid line is a bicomponent fit.

strength. In each case, the water layer is to the left, the cream layer to the right. The topmost profiles were recorded with near zero diffusion gradient, and faithfully reflect the ${ }^{1} \mathrm{H}$ distribution as measured in the $T_{1}$ experiments-compare Figs. 3 and 5(a), for instance. The lowest profiles were recorded with maximum diffusion gradient and are substantially different in shape both from the top profile and between the emulsions. The water signal is completely attenuated. The cream signal is attenuated much less than in bulk decane (not shown), and this is attributed to restricted diffusion in the droplets. The attenuation clearly depends both on position within the emulsion and the gum xanthan content.

Figure 6(a) shows a typical echo signal attenuation curve for a water layer, this example taken from the center of the water layer in emulsion $E 0$. The corresponding fit according to Eq. (2) is also shown from which the water self diffusion coefficient is calculated to be $2.11 \times 10^{-5} \mathrm{~cm}^{2} \mathrm{~s}^{-1}$, in excellent agreement with accepted values in the literature [22].

For all the experiments reported here, the long time limit is generally well met for the droplets and the data within the cream layer have been analyzed accordingly. The signal-tonoise ratio of the profiles is insufficient to allow an independent multicomponent fit to the data at every location, without some constraint being placed on the droplet size distribution. Therefore, the analysis presented here has been carried out using the result due to Ref. [20], [Eq. (10)], which, as stated assumes a Gaussian distribution of droplet sizes at every location. Where appropriate, analysis according to the Murday and Cotts formalism has also been carried out and, although not reported here, found to be in broad agreement.

In many cases, a unimodal Gaussian distribution of droplet sizes has been sufficient to obtain an adequate fit to the experimental data. As an example to validate the general procedure, consider Fig. 6(b), which shows representative echo attenuation data recorded from the cream layer of emul- 


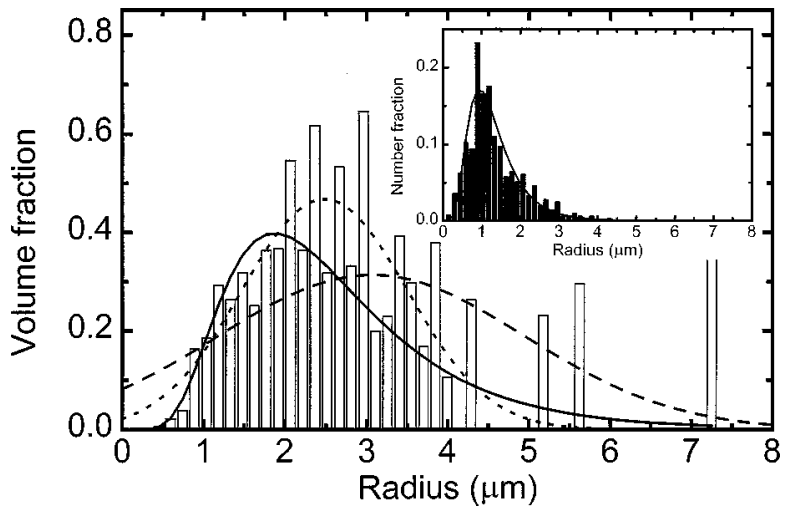

FIG. 7. The inset shows the optically measured droplet size distribution for emulsion Ehom. The solid line is a log-normal fit to the data $\left(r_{0}=1.18 \mu \mathrm{m}, \sigma=0.48\right)$. The histogram and solid line in the main figure are the corresponding volume distributions. The dotted line is a Gaussian fit to the volume data $\left(r_{v 0}=2.48 \mu \mathrm{m}, \sigma_{v}\right.$ $=1.00 \mu \mathrm{m}$ ). Finally, the dashed line is the (averaged) Gaussian volume distribution obtained from the PGSE profiling data such as in Fig. 6(b).

sion Ehom (there is little spatial variation). This emulsion contains a unimodal, narrow distribution of small droplets. The solid line in the figure is the best fit to the data according to Eq. (10). The fit parameters are $r_{\nu 0}=3.06 \mu \mathrm{m}$ and $\sigma_{v}$ $=1.87 \mu \mathrm{m}$. Also shown in the figure by dashed lines are secondary fits in which the key parameters, the mean droplet radius and distribution width, have been systematically varied by $10 \%$ and $30 \%$, respectively. These fits are noticeably worse and therefore yield an estimate of the errors in the best fit parameters. The oil droplet size distribution measured by NMR is in good agreement with the separate optical analysis of this emulsion. The inset of Fig. 7 shows the measured droplet size (number) distribution and associated log-normal fit, with fit parameters $r_{0}=1.18 \mu \mathrm{m}$ and $\sigma=0.48$. The main figure shows the corresponding droplet size volume distribution together with the corresponding fit calculated from the aforementioned log-normal distribution (solid line). Also shown are a Gaussian fit to the volume distribution data $\left(r_{v 0}=2.48 \mu \mathrm{m}, \sigma_{v}=1.00 \mu \mathrm{m}\right.$, dotted line) and the Gaussian distribution based on the NMR result (dashed line). There is no reason a priori for choosing either the log-normal number distribution or the Gaussian volume distribution except that, for the number distribution, the log normal is common practice, and for the PGSE NMR, the Gaussian is a tractable calculation. A further example of a unimodal data fit, this time to a slice at the center of the cream layer in the (bimodal) emulsion E0 is shown in Fig. 6(c).

In other cases, a unimodal droplet size distribution is insufficient to interpret the data. As an example, consider Fig. 6(d), which contains data from the upper part of the cream layer in the emulsion with $0.1 \%$ xanthan, E100. The dotted line is the "best"' fit using a unimodal distribution. The solid line is a fit using a bimodal distribution and clearly much better reproduces the data. The fit parameters are $r_{\nu 0}^{(1)}=2.87$ $\mu \mathrm{m}$ and $\sigma_{v}^{(1)}=2.06 \mu \mathrm{m}(36 \%)$ and $r_{\nu 0}^{(2)}=14.3 \mu \mathrm{m}$ and $\sigma_{\nu}^{(2)}$ $=23.0 \mu \mathrm{m}(64 \%)$. There are, of course, instances where a subjective judgment is required as to whether a unimodal or bimodal distribution is better. Where this is ambiguous, a

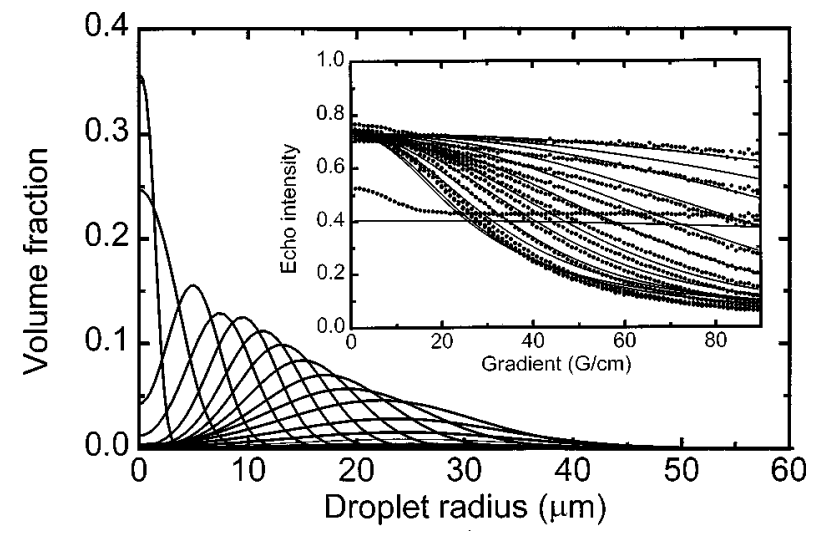

FIG. 8. The droplet size distribution of emulsion E0 as calculated from the NMR data as a function of position in the cream layer. The most intense and narrowest distribution occurs at the base of the cream layer, and the curves proceed logically up through the cream layer in steps of $0.041 \mathrm{~cm}$. The data should be compared with the prediction of the model of Ref. [5], Fig. 13. The inset makes the same comparison in $\mathbf{q}$ space. It shows the PGSE data recorded at each position (top trace is equal to the bottom of the cream layer) compared with the predictions of the model evaluated as discussed in the text. The curious horizontal trace in the center of the plot is due to partial volume filling at the water-cream interface, and includes the initial, rapid, water decay in the experimental data.

single mode distribution is chosen in an effort not to overinterpret the data.

The main parts of Figs. 8 and 9 show the droplet size distribution as a function of height in the cream layer as calculated from the NMR data according to the foregoing analysis for emulsions $E 0$ and $E 100$, respectively. With no xanthan (Fig. 8), there is a strong separation of small and large droplets according to height. This separation continues with increasing xanthan concentration but becomes noticeably less marked. In the emulsion with $0.1 \%$ xanthan, $E 100$ (Fig. 9), the droplet size distribution is more or less constant as a function of height, with both small and large droplets found at each level. These differences and the insets to the figures are discussed further in Sec. V.

PGSE profiles have also been recorded with the diffusion gradient oriented along the vertical $z$ direction as well as the horizontal $x$ direction (Fig. 10). In this way the measurement

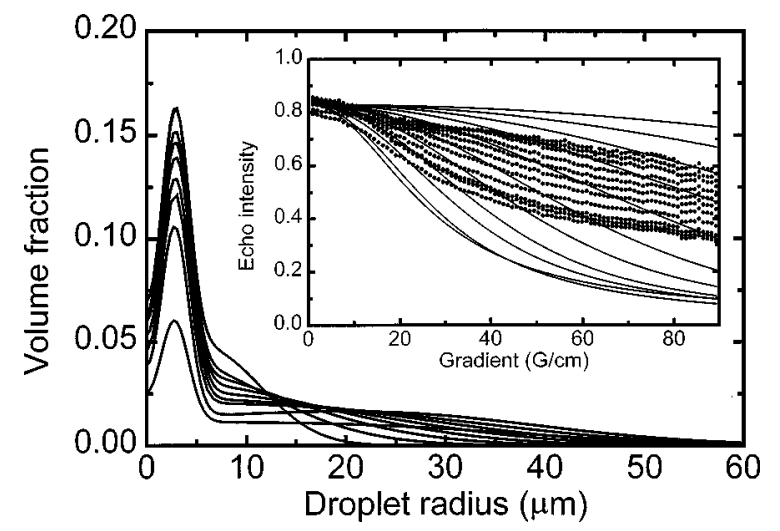

FIG. 9. As in Fig. 8, but for emulsion E100. Comparison either in real space (i.e., with Fig. 13) or $\mathbf{q}$ space (inset) shows that the model fails for this emulsion. 


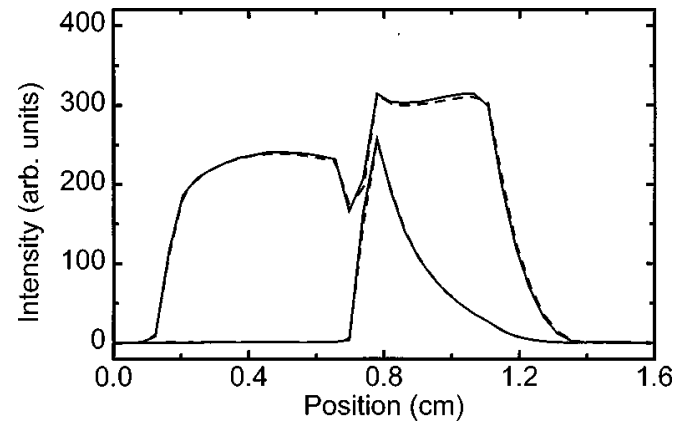

FIG. 10. A comparison of the PGSE attenuated profiles for emulsion E33 recorded with gradient strengths of 0.95 (upper traces) and $91.2 \mathrm{G} / \mathrm{cm}$ (lower traces), and the pulsed field gradient oriented along horizontal (solid lines) and vertical (dashed lines) axes. The very high degree of correspondence between the traces suggests very little droplet anisotropy.

is made sensitive to the critical droplet dimension in the two different orientations. Any differences would indicate a departure of the droplet shape from spherical toward ellipsoidal. However, an extremely close correspondence of the data has been seen for all gradient strengths and for all positions in the emulsion examined in this way (E33). This experiment therefore suggests that the droplets are spherical, or at least not ellipsoidal, to well within the experimental accuracy of the measurement. The method is not sensitive to near isotropic deformations such as with randomly oriented polyhedra.

Figures $11(\mathrm{a})$ and $11(\mathrm{~b})$ are the corresponding ${ }^{13} \mathrm{C}$ edited versions of Figs. 5(a) and 5(b), respectively. Only the oil in the cream layers is seen. The uppermost traces recorded with near-zero PGSE gradient no longer have the square shape associated with the ${ }^{1} \mathrm{H}$ measurements. This is attributed, more than anything else, to spatial variation in spin relax-

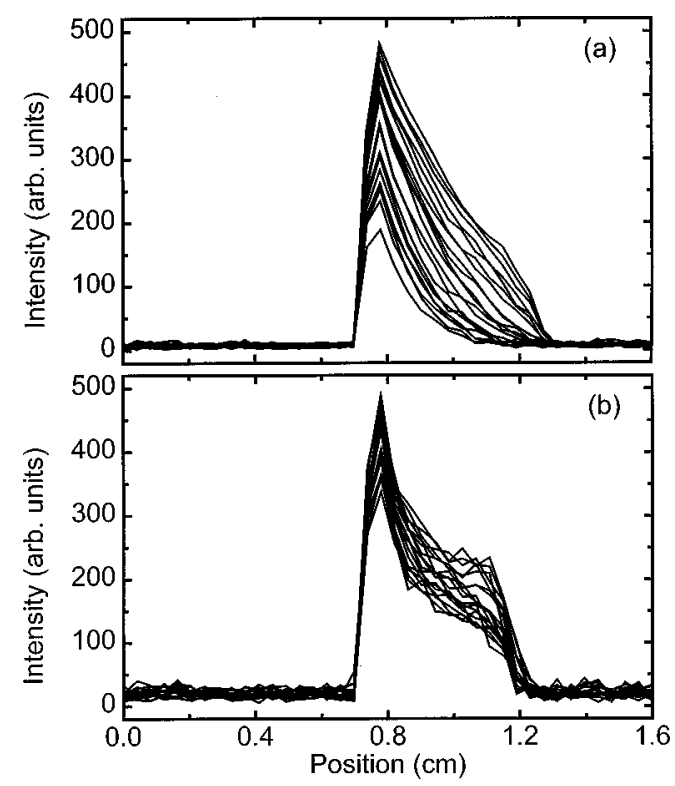

FIG. 11. (a) PGSE weighted, ${ }^{13} \mathrm{C}$ edited profiles of the creamed emulsion E0. From the top, the profiles are shown for gradient strengths from 1.42 to $86.92 \mathrm{G} / \mathrm{cm}$ in steps of $4.275 \mathrm{G} / \mathrm{cm}$. (b) PGSE weighted, ${ }^{13} \mathrm{C}$ edited profiles of the creamed emulsion $E 100$. From the top, the profiles are shown for gradient strengths from 4.8 to $91.2 \mathrm{G} / \mathrm{cm}$ in steps of $4.8 \mathrm{G} / \mathrm{cm}$.

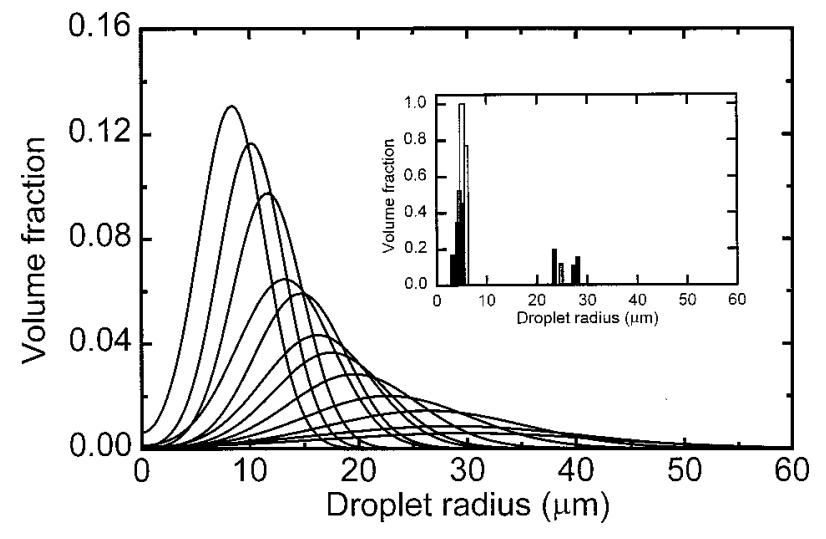

FIG. 12. As the main part of Fig. 8 and for emulsion $E 0$, but evaluated from the ${ }^{13} \mathrm{C}$ edited data. The inset is for $E 100$. The lighter shading corresponds to the base of the cream layer. The ${ }^{13} \mathrm{C}$ edited results support the conclusions of the ${ }^{1} \mathrm{H}$ NMR.

ation times and radio frequency pulse imperfections arising from excitation field inhomogeneity, and therefore to spatial variation in the cross-polarization transfer efficiency. A correction for relaxation losses can be made for relaxation time variation across the sample on the basis of the measurable $T_{1 \rho}$, the spin-lattice relaxation time in the rotating frame (see Ref. [12]). We have carried out an approximate correction by setting $T_{1 \rho}=T_{2}$ of the protons, and found that the profiles are much more rectangular. However, caution must be applied since, in reality, the relaxation rate is unlikely to be unimodal but rather will reflect the droplet size distribution. Notwithstanding these comments, the ${ }^{13} \mathrm{C}$ edited profiles provide complementary evidence that the conclusions drawn from the ${ }^{1} \mathrm{H}$ profiles are largely correct, and that the signal from water in the cream layer in the ${ }^{1} \mathrm{H}$ profiles is small, well attenuated, and does not significantly affect the results.

Figure 12 shows the droplet size distribution for emulsion $E 0$ and, in the inset, for emulsion $E 100$ derived from the ${ }^{13} \mathrm{C}$ edited profiles. The former emulsion contains enriched toluene, and a good signal-to-noise ratio is therefore available. In the case of emulsion $E 100$, which had no enriched toluene, it has only been possible to fit the data according to the unimodal and bimodal versions of the Tanner and Stejskal result [Eq. (7)], and thus the distributions have no width. However, for both emulsions, the general trends are the same as for the ${ }^{1} \mathrm{H}$ profiles, and support the contention that any residual water in the cream layer, save at the very base where it is explicitly observed and accounted for, is having a negligible effect on the data. Presumably this is because the water layer is not confined, and its signal is thus rapidly attenuated to zero.

Before the differences in the droplet size distribution with position in the cream layer are discussed further and related to the modeling of the creaming process, some limitations to the data fitting must be acknowledged. As Callaghan, Jolley, and Humphrey pointed out [20], Eq. (10) strictly only applies when $r_{\nu 0} \gg \sigma_{v}$; otherwise a significant number of droplets with "negative radii" are predicted. This is a condition not always fulfilled in this work. However, since all the results depend on $r^{2}$, we suggest that it is acceptable to fold the calculated droplet distribution inferred with a negative radius back into the corresponding positive radius distribution and 


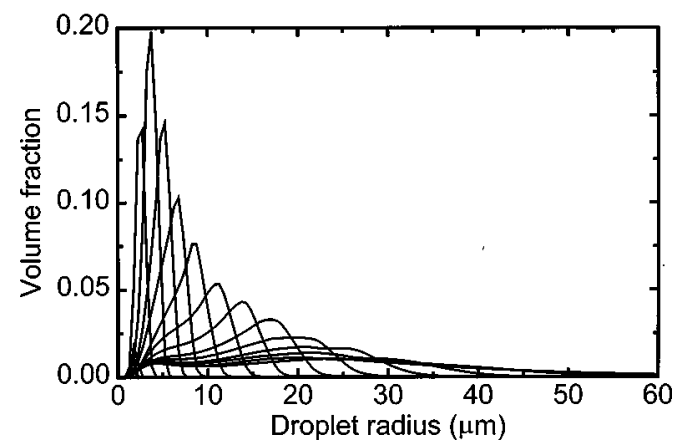

FIG. 13. The droplet size distribution for emulsion $E 0$ as evaluated according to the model of Ref. [5]. The traces with maxima proceeding from left to right range from the bottom to the top of the cream layer in step sizes directly comparable with the magnetic resonance profiles. The predictions for emulsion E100 are not significantly different.

to accept the resulting (non-Gaussian) distribution as being characteristic of the emulsion. We have affirmed the validity of this approach by calculating a Gaussian distribution from an echo decay train [Eq. (10)], carrying out the folding procedure as described, and, from the resulting distribution, have recalculated the expected NMR signal using the Tanner-Stejskal formula for each separate droplet size $r$ prior to summing over all sizes. The data was faithfully reproduced-moreso indeed than when the folding step was not included. Clearly when $r_{v 0} \ll \sigma_{v}$, the model predicts an infinite number of droplets with zero radius and volume. Situations where this occurs, notably at the very base of some cream layers, are self evident, and the interpretation should in these instances be treated with caution. Moreover, in these situations, the fit tends to be insensitive to the $r_{\nu 0}$ parameter since the distribution width is relatively large. In such cases, data recorded with other timing parameters which are more sensitive to the small droplets have been of considerable assistance. Finally, toward the bottom of the cream layer, the data often show evidence of a mobile component which is taken to be water. Here, it is clearly justifiable to fit the data to two components-one according to Eq. (10) representing the oil droplets, and one according to Eq. (2) representing the continuous water component.

\section{MODELING AND DISCUSSION}

Figure 13 shows the droplet size distribution as a function of position in the cream layer of an emulsion calculated according to the model of Ref. [5]. The model emulsion is based on the known physical parameters of decane, toluene, and water, on the measured droplet size distribution expressed in Fig. 1, and on a critical cream concentration of $73 \%$, determined from the widths of the cream and water layers in the emulsion and the known composition. It is clear that this plot has many characteristics similar to Figs. 8 and 12 , the interpreted data for the emulsion with no gum xanthan. It is equally evident that it has little in common with Fig. 9 and the inset to Fig. 12, the interpreted data for the emulsion with most gum xanthan.

For the xanthan rich sample, only three variables (excluding density differences due to the toluene fraction, of which account has been taken) are available to change in the model

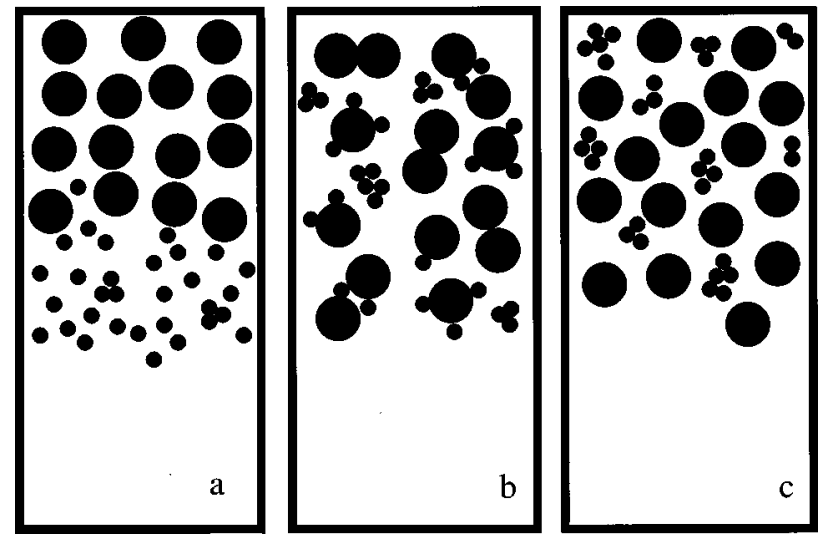

FIG. 14. A schematic representation of the cream layer as suggested by this work (a) without and [(b) and (c)] with 0.1 -wt \% gum xanthan dissolved in the water. In (b), only depletion flocculation is considered. In (c), depletion stabilization is considered as an additional mechanism.

to try and achieve a better fit to the measured data. These are the critical cream concentration, the droplet size distribution, and the viscosity. The viscosity varies with the xanthan concentration, but, according to the model, has no effect on the final droplet size distribution. The droplet size is known not to vary too greatly between the emulsions. There is evidence that the cream layer is denser in the $0.1 \%$ xanthan emulsion, of the order of $83 \%$. However, this does not change the modeled distribution significantly.

To see how great the differences are, and to exclude any artifact of the data fitting procedures, we compare model and data not in real space, but in $\mathbf{q}$ (measurement) space. That is, for each droplet size we calculate the echo attenuation using the Tanner-Stejskal result, and, for the modeled droplet size distribution, sum over all droplet sizes. The results of this comparison are shown in the insets to Figs. 8 and 9. Again, it is clear that the model corresponds well to the data for the emulsion with no gum xanthan. There is a clear differentiation of droplet size with position. This is not the case for the emulsion with $0.1 \%$ gum xanthan. The differences are not due to the presence or absence of toluene in the two emulsions presented here: a transition from spatially dependent to independent droplet size distribution is seen throughout the emulsion series $E 0, E 3, \ldots, E 100$ which variously contain toluene.

The variation of droplet size distribution with gum xanthan content is attributed to the effects of depletion flocculation and depletion stabilization. With no gum xanthan in the emulsion, depletion flocculation and stabilization are not mechanisms to be considered. Under these circumstances, the model due to Ref. [5] reasonably describes the dynamic processes taking place during creaming. Large droplets are found at the top of the cream, small droplets at the base, a situation shown schematically in Fig. 14(a). With the addition of gum xanthan, flocculation is important [2,3]. At $0.1 \%$ xanthan concentration in the continuous component, it is possible that droplets randomly aggregate into flocs containing a distribution of droplet sizes. These flocs all have a similar effective size, and hence rise into the cream layer at a similar rate. However, as noted earlier, the potential barrier to flocculation and the depth of the potential minimum vary 
with the drop radius [6] and, as a result, depletion stabilization may also be important in the xanthan rich emulsion. Thus it may be that small droplets flocculate while larger droplets are depletion stabilized. Under these circumstances, the flocs of small droplets have an effective radius comparable to the larger droplets. Again all droplets rise into the cream layer at a similar rate. In either case, the result is a uniform distribution of droplet sizes through the cream layer. The alternate scenarios are depicted schematically in Figs. 14(b) and 14(c).

According to recent Monte Carlo [23] simulations of forces between colloidal particles in polymer solutions, depletion stabilization is a less likely mechanism than depletion flocculation in our system of study. It was found that the force between particles is purely attractive in semidilute solutions. Repulsive forces, which enable depletion stabilization, operate at higher concentrations. On this basis, the scenario in Fig. 14(b) is the more likely. To verify this requires further experimental results. It is now important to gain data on the creaming rates and the time dependence of the spatial distribution of droplets for emulsions with very narrow droplet size distributions with carefully controlled fractions of well characterized polymer in the continuous component. That NMR can provide this information is now established.

Similar q space comparisons of data recorded immediately after the development of the cream layer, when creaming is first judged complete, typically at $0.5-5$ days dependent on xanthan concentration, and much later might be expected to reveal any redistribution of the droplets within the cream layer over time. No evidence for any such redistribution has been obtained.

\section{CONCLUSIONS}

There are two primary conclusions to be drawn from this work. The first is that a combination of NMR imaging and pulsed-field-gradient spin-echo-restricted diffusometry is a reliable means of gaining the spatial dependence of the droplet size distribution in the cream layer of a turbid emulsion. For this purpose, ${ }^{13} \mathrm{C}$ edited CYCLCROP pulsed-fieldgradient diffusometry has been introduced as a technique. The overriding advantage of this method is that the water signal is totally suppressed. The disadvantages, however, are twofold. First, the natural abundance of ${ }^{13} \mathrm{C}$ leads to a low signal-to-noise ratio in the experiment unless ${ }^{13} \mathrm{C}$ enriched material is used. For this reason, ${ }^{13} \mathrm{C}$ enriched toluene mixed with the decane was used in some of this work. The second is that the magnetization transfer is a relatively time consuming process, and here takes a time of the order of the oil $T_{2}$, so that the ${ }^{13} \mathrm{C}$ edited signal is partly attenuated. Moreover, the cross-polarization sequence used in these experiments is highly frequency selective, and therefore background field gradients can lead to a locally reduced transfer efficiency.

The second conclusion is that the droplet size distribution in a simple oil-in-water emulsion varies with position in the cream layer in a manner dependent on gum xanthan concentration. This dependence is not fully accounted for by current theory, but is broadly explicable if depletion flocculation and depletion stabilization are considered as active mechanisms. To our knowledge, this is the first time that this spatial dependence has been unambiguously demonstrated.

\section{ACKNOWLEDGMENTS}

P.J.M. thanks the Alexander von Humboldt Foundation for financial support. E.C. thanks the U.K. Engineering and Physical Sciences Research Council for financial support. The authors thank Dr. E. Sautter (Universität Ulm) for assistance with the optical microscopy.
[1] R. J. Hunter, Foundations of Colloid Science (Clarendon, Oxford, 1987), Vol. 1.

[2] E. Dickinson and S. R. Euston, in Food Polymers, Gels and Colloids, edited by E. Dickinson (Royal Society of Chemistry, Cambridge, 1991).

[3] D. J. Hibberd, A. M. Howe, A. R. Mackie, P. W. Purdy, and M. M. Robins, in Food Emulsions and Foams, edited by E. Dickinson (Royal Society of Chemistry, London, 1987).

[4] S. Asakura and F. Oosawa, J. Polym. Sci., 33, 183 (1958).

[5] V. J. Pinfield, E. Dickinson, and M. J. W. Povey, J. Colloid Interface Sci. 166, 363 (1994).

[6] R. I. Feigin and D. H. Napper, J. Colloid Interface Sci. 75, 525 (1980).

[7] P. Jokela, P. D. I. Fletcher, R. Aveyard, and J. R. Lu, J. Colloid Interface Sci. 134, 417 (1990).

[8] B. Newling, P. M. Glover, J. L. Keddie, D. M. Lane, and P. J. McDonald, Langmuir 13, 3621 (1997).

[9] K. J. Packer and C. Rees, J. Colloid Interface Sci. 40, 206 (1972).

[10] I. Lönnqvist, B. Hakansson, B. Balinov, and O. Söderman, J.
Colloid Interface Sci. 192, 66 (1997).

[11] C. Kunze and R. Kimmich, J. Magn. Reson., Ser. B 105, 38 (1994).

[12] M. Heidenreich, W. Köckenberger, R. Kimmich, N. Chandrakumar, and R. Bowtell, J. Magn. Reson. 132, 109 (1998).

[13] N. Chandrakumar and R. Kimmich (unpublished).

[14] E. O. Stejskal and J. E. Tanner, J. Chem. Phys. 42, 288 (1965).

[15] J. E. Tanner, J. Chem. Phys. 52, 2523 (1965).

[16] P. T. Callaghan, Principles of Nuclear Magnetic Resonance Microscopy (Clarendon, Oxford, 1991).

[17] J. S. Murday and R. M. Cotts, J. Chem. Phys. 48, 4938 (1966).

[18] J. E. Tanner and E. O. Stejskal, J. Chem. Phys. 49, 1768 (1968).

[19] P. T. Callaghan, J. Magn. Reson. Ser. A 113, 53 (1995).

[20] P. T. Callaghan, K. W. Jolley, and R. S. Humphrey, J. Colloid Interface Sci. 93, 521 (1983).

[21] J. M. Ziman, Models of Disorder (Cambridge University Press, Cambridge, 1979).

[22] R. Mills, J. Chem. Phys. 77, 685 (1973).

[23] R. Dickman and A. Yethiraj, J. Chem. Phys. 100, 4683 (1994). 